Type of the Paper (Article)

\title{
Migration of Human Bone Marrow-Derived Mesenchymal Stem Cells during Wound Healing
}

\author{
J. Michael Sorrell 1, Rodrigo A. Somoza 2, Marilyn A. Baber ${ }^{3}$, Jonathan Kenyon 4, and Arnold I. Caplan 5*
}

${ }^{1}$ Skeletal Research Center, Department of Biology, Case Western Reserve University, Cleveland OH, USA 44106;

\author{
jms30@case.edu \\ 2 Skeletal Research Center; ras286@case.edu; 3 Skeletal Research Center; mababer844@aol.com; 4 Skeletal \\ Research Center; jdk39@case.edu; 5 Skeletal Research Center; aic@case.edu \\ * Correspondence: e-mail@e-mail.com; Tel.: (optional; include country code; if there are multiple correspond- \\ ing authors, add author initials)
}

\begin{abstract}
Bone marrow derived adult human mesenchymal stem cells (hMSCs) possess therapeutic qualities that enable them to enhance wound repair. However, the mechanisms by which this occurs remains poorly understood. Basic mechanisms may include the directed migration of delivered cells to target sites and/or the production and release of soluble factors that act at a distance. Allogeneic and even xenogeneic cells may effectively participate in wound repair. Labeled hMSCs were delivered to full-thickness skin wounds that were created in immunologically competent mice. The delivery occurred on day 3 post-wounding using two different carriers; one which released cells and one which retained cells. The fates of the delivered cells were tracked for up to 25 days. During this period, released cells migrated as a tight cohort deep into the wound to reach the subdermal vascular plexus. Simultaneously, enhanced formation of granulation tissue was evident. This migration of hMSCs was not essential in that enhanced granulation tissue formation and wound contraction occurred when cells were retained in the carrier matrix. This provided further evidence for the release of therapeutic factors by hMSCs to sites of injury.
\end{abstract}

Keywords: Mesenchymal Stem Cells 1; Cell Migration 2; Xenogeneic Wound Repair 3.

\section{Introduction}

Human marrow-derived mesenchymal stem cells (hMSCs) survive and promote wound repair in fully immunocompetent mice. This feature is consistent with the notion that these cells possess immunomodulatory characteristics that enable them to promote wound repair in allogeneic and xenogeneic situations [1-7].

The initial resolution of deep cutaneous wounds involves a cascade of cellular responses that are necessary to prevent infection and blood loss [8]. Subsequently, a neo-vascularized wound tissue develops and the wound closes $[9,10]$. In rodents, vascular restoration is initiated at the base of the wound from the subdermal vascular plexus and wound closure is simultaneously mediated by the panniculus carnosum, which is also located at the base of the wound, 
beneath the vascular plexus [11-14]. In this study, adult human mesenchymal stem cells (hMSCs) upon delivery by carriers targeted both vasculature development and wound closure in normal mice.

The fates of labelled hMSCs were determined by imaging tissue sections at timed intervals. The hMSCs that were freed from their carrier migrated over a period of several days to the subdermal vascular plexus. During this period enhanced granulation tissue formation and wound closure was observed. Labelled cells that were retained in their carrier matrix did not migrate. However, these cells still promoted enhanced vascularity and wound closure compared with control matrix without cells. Thus, hMSCs affect wound healing events at a distance, but when free to do so migrated. These results indicate that hMSCs remain viable and capable of migrating in the context of a wound environment. Further, these data indicate that products secreted by hMSCs alter distant targets and promote wound repair.

A murine cutaneous wound repair model was developed to investigate these issues. Standard protocols in wound repair studies call for the delivery of potential therapeutic cells at the time of injury [1,6,15,].

However, this may not be the most efficient approach because delivered cells may diffuse in a large wound limiting effectiveness. Preliminary studies indicated murine full-thickness cutaneous wounds when well bandaged form a cellular but avascular wound bed at the wound site by the third day. This wound bed has the potential for providing a substrate that facilitates the entrance of hMSCs into the wound bed. Thus, a more effective approach in this model is delivery of hMSCs to the surface of this wound bed on day 3 post-wounding. This method allows us to evaluate the effects of hMSC migration and secretion of soluble factors on wound healing.

Equivalent numbers of labelled MSCs were delivered to wound sites in two types of hyaluronan-based scaffolding: Glycosil [16] and Hyalomatrix PA [17,18]. The Glycosil scaffolds released cells while Hyalomatrix scaffolds retained cells. This allowed the effect of cellular migration of unrestricted cells and paracrine factors released by immobilized cells in wound repair to be determined.

\section{Materials and Methods}

Antibodies. Rat anti-mouse CD31 was purchased from Millipore/Chemicon as was the appropriate fluorescein isothiocyanate (FITC)-conjugated second antibody. This antibody preparation is specific for mouse vasculature. The vital dye CM-DiI was purchased from Molecular Probes/Life Technologies (Grand Island, NY).

Cells. The study was conducted according to the guidelines of the Declaration of Helsinki, and approved by the Institutional Review Board. Human adult bone marrow derived hMSCs were obtained from the Hematopoietic Stem Cell Facility of Case Western Reserve University and University Hospitals under IRB protocol UH IRB 09-90-195. Informed consent was obtained from all bone marrow donors. Bone marrow aspirates were brought into the laboratory and hMSCs were established from the adherent cell populations as previously described without added fibroblast growth factor-2 [19]. The hMSCs from a single human donor were employed throughout this study. This set of hMSCs was selected for their ability to support vascular tubule formation in culture, an indication that they were producing pro-angiogenic factors [20]. Cells from passages 2-4 and were labeled with CM-DiI as previously described [20].

\section{Murine Cutaneous Wound Model}

Wound Creation. All procedures were performed in the Case Animal Resource Center using procedures that were approved by the Case Western Reserve University Institutional Animal Care and Use Committee (protocol 2008-0132). Male Balb-cJ mice (Jackson Laboratories, Bar Harbor, ME) that were 2-3 months old were used for all applications. Anesthetized mice were shaved and an 8-mm area of skin was marked and lifted so that scissors could cut an 8-mm full-thickness wound. The wound was immediately covered with Vaseline gauze (Covidien, Mansfield, MA), Tegaderm ${ }^{\mathrm{TM}}$ (3M Critical and Chronic Care Solution, St. Paul, MN), and an adhesive bandage (Figure 1). This wound 
coverage inhibited desiccation of the wound and scab formation. Each mouse was placed in a separate cage at this point. On day 3 , the mice were anesthetized and the bandages and wound coverings were removed. Scab formation on day 3 was rare, but if present, it was gently removed. However, mice with damaged wounds at this point were excluded from the study. Hyaluronan-based carriers, either seeded with cells or with no cells (control), were placed onto the wound surface with the cell-side down. The wounds were again covered with Vaseline gauze, Tegaderm (now attached with sutures at each of the four corners), and an adhesive bandage. Mice were returned to separate cages. The earliest time-point for sacrifice was day 3 post-application of cells. All bandages were required by the protocol to be removed no later than day 7 post-application of cells. By this time, hMSCs had become engrafted into the wound bed. Mice were sacrificed at various time-points up to day 25 post-application of cells. A total of 90 mice were used for the study: 45 received Glycosil + hMSCs, 20 received Glycosil alone, 15 received Hyalomatrix PA + hMSCs, and 10 received Hyalomatrix PA alone.

Hyaluronan Delivery Vehicles. Two hyaluronan-based matrices were used for this study. Glycosil ${ }^{\mathrm{TM}}$ was provided by Dr. Glenn Prestwich [16] and Halomatrix PA ${ }^{\mathrm{TM}}[17,18]$ was provided by Dr. Alessandra Pavisio, Fidia Advanced Biopolymers, now a division of Anika Therapeutics (Bedford, MA). These carriers were sterile upon arrival and sterility was maintained during cell application through delivery to the wound. Glycosil disks were fabricated from polyethyleneglycol tetraacrylate-crosslinked thiol-modified hyaluronan (Glycosan BioSystems, Salt Lake City, UT). This consists of a meshwork of fine fibrils prepared as 6-mm disks. Hyalomatrix PA, a United States Federal Drug Administration approved wound healing product, consists of thick woven hyaluronan fibers that are attached to a semipermeable silicone backing. This material was cut in to disk using a sterile 6-mm biopsy punch in order to insure its fit into an 8-mm wound. The hydrophobicity of the matrix was reduced by pre-treatment with medium that contained $10 \%$ fetal bovine serum overnight at $4^{\circ} \mathrm{C}$. The $6-\mathrm{mm}$ disks were seeded $50 \mu \mathrm{l}$ of medium that contained 250,000 cells approximately 2-5 hours prior to their placement onto the wound surface. Control disks were seeded with an equal volume of medium without cells. Tests were performed to assure viability of cells over 3 days.

Wound Analyses. Wounds areas and surrounding tissue were excised from euthanized mice at various time points starting on day 3 post-application and lasting until day 25 post-application. The wounds were photographed prior to and after their excision. The bottom surface of a rodent wound provides critical information regarding vascularization and contraction, therefore it was also photographed. Unfixed wounds were placed in OCT-embedding compound (Electron Microscopic Sciences, Hatfield, PA) and frozen in liquid nitrogen. Sections of $10 \mu \mathrm{m}$ thickness were cut through the wound and mounted onto slides for viewing using combined phase contrast and fluorescent optics. This provided a means to visualize CM-DiI labeled cells and to determine the location of these cells within the wound bed. Unstained sections were photographed and selected sections were immunostained with a rat-anti-mouse antibody specific for CD31. The sections were then treated with a second antibody conjugated with FITC. Other selected sections were stained with hematoxylin and eosin. All photography for sectioned material was performed on a Leica microscope equipped with a digital camera. RGB-images were saved in TIFF-format. Exposure intensity was adjusted using Adobe Photoshop CS4 and composite images were created using Adobe Illustrator CS3.

\section{Results}

\subsection{Wound Creation}

Murine skin wounds were created as described in the Methods section. The standard procedure for wound intervention calls for the delivery of potentially therapeutic cells at the initial time of wounding [2,6,7,11,15]. Histological analyses of early wounds found that a well bandaged wound began to form an avascular, but cellular wound bed by day 2 post-wounding (Figure 2). Therefore, it was possible to remove the bandage on day 3 and apply hMSCs in carriers directly to this wound bed (Figure 3). This proved to be a more effective delivery approach. Two 
carriers were selected for this purpose. The Glycosil carrier delivered cells, but then rapidly disintegrated, thus releasing cells to the wound bed (Figure 4). The Hyalomatrix PA carrier remained intact and did not release enmeshed hMSCs. Both methods were successful in the delivery of functional hMSCs to skin wounds. In vitro cell viability studies of cell seeded scaffolds indicated hMSCs survived in the scaffold for at least 3 days (data not shown).

\subsection{The Fate of Delivered hMSCs by Glycosil}

The CM-DiI labelled hMSCs delivered by Glycosil were tracked in unstained frozen sections of wound tissues that were taken at multiple time points following their application to the wound (Figure 5). Applied hMSCs migrated deep into the wound bed and eventually entered the subdermal vascular plexus by day 17 post-delivery. There was no indication that hMSCs physically associated with the granulation tissue vasculature during their passage through this tissue. However, upon arrival in the subdermal vascular plexus, hMSCs and mouse vasculature appeared to be closely integrated (Figure 6).

The therapeutic effects of hMSCs were demonstrated by the early appearance of granulation tissue and early wound closure compared with Glycosil carrier alone controls (Figure 7). The wounds receiving hMSCs contracted laterally and brought in undamaged dermis containing hair follicles. This implies enhanced restoration and contraction of the panniculus carnosum muscle [11,13]. In contrast, control wounds contained a poorly organized vasculature network.

\section{3. hMSC Migration}

The results presented above indicate that hMSCs have the capacity to migrate in wound environments. By the end of our experiment, hMSC homed to the subdermal vascular plexus suggesting this vasculature directs injury induced hMSC migration. This concept is further supported by in vitro studies in which hMSCs migrate towards and co-align with HUVEC generated vascular tubes [20]. It was not possible in the current study to interrogate the hMSC transcriptome in the wound bed. However, the genomic interrogation of cultured hMSCs reveals information regarding migration potential. Previously RNA-seq genomic profiles were generated from three different donor populations in order to assess expression of genes related to vascular migration [21]. Vascular endothelial cell controlled migration is in large part directed by vascular endothelial growth factor receptors-1 and -2 [22,23]. Transcriptome analyses of hMSCs demonstrated these cells lack expression of both VEGFR1 and VEGFR2 (Table 1). Vascular endothelial cells also express alternate receptors known to enhance migration and form tube-like structure during vasculogenesis. Two of these alternate receptors are the neuropilins-1 and -2 [24]. In contrast to the principal VEGF receptors, cultured hMSCs express NPR1 and NPR2. These receptors interact with multiple ligands including semaphorins and multiple VEGFs [25,26]. In addition, hMSCs also express the genes for hepatic growth factor receptors and platelet derived growth factor receptors (Table 1), both critical for angiogenesis [27-29].

Wound data indicate that delivered hMSCs promote granulation tissue formation and in vitro studies support this by indicating hMSCs support vasculature tubule formation and assume a perivascular orientation [20,30]. This implies hMSCs release pro-angiogenic factors. The RNA-Seq genomic profile of cultured hMSCs provides evidence hMSCs express genes for VDGF-A, angiopoitin-1, angiopoitin-like-2, and HGF (Table 1b). These are all soluble proangiogenic factors [31].

\subsection{The Fate of Delivered hMSCs by Hyalomatrix PA}

Hyalomatrix PA carriers with and without hMSCs were delivered to murine wounds on day 3 post wounding in the same manner as Glycosil carriers. However, the Hyalomatrix carriers remained intact and did not release cells 
during the healing process. The differences between matrices with and without cells is shown in Fig 8. The presence of hMSCs resulted in enhanced production of granulation tissue. The engrafted Hyalomatrix PA contained both labeled hMSCs and mouse vasculature. No vasculature was present in the matrix in the absence of hMSCs. The bottom surfaces of skin wounds also showed increased vascularity and wound contraction when hMSCs are present in the wound. These results again provide evidence hMSCs release factors promoting wound healing at a distance.

\section{Discussion}

The promotion of wound repair by xenogeneic hMSCs in immunocompetent mice provides evidence for their strong immunomodulatory characteristics. The therapeutic application of hMSC cells and/or conditioned media to promote wound healing has been shown in multiple studies $[1,6,7,14,32-34]$. In many, the therapeutic agents were delivered directly to the wound at the time of injury. The current study proposes the optimal delivery time occurs on day 2 to 3 post-wounding. At this stage, a cellular but avascular wound bed appears when wounds are well bandaged. Without bandaging, a scab forms which is difficult to remove without further damage to the wound. This wound bed provides a substantial support matrix through which cells can migrate as a concentrate cohort.

These studies provide conclusive evidence hMSCs migrate into and through a wound environment. The migration of hMSCs targeted and integrated into the subdermal vascular plexus, suggesting these cells are attracted to this vascular network. The attraction of hMSCs to vasculature has been demonstrated in other studies. hMSCs reportedly belong to a category of perivascular cells termed pericytes [35]. Pericytes are recruited to the microvasculature by chemokines such as PDGFs $[28,36]$. Others have shown that in vivo engraftment of MSCs occur at sites of injury where they present a perivascular orientation $[37,38]$.

In culture studies, randomly seeded hMSCs migrated to and co-aligned with vascular tube-like structures [20]. The mechanism driving this migration are not fully understood. However, cultured hMSCs express genes for PDGFs and PDGF receptors [21]. However, other factors may also play a role in the directed migration of hMSCs. As indicated in this study, these cells do not express the principal VEGF receptors, but they do express alternate VEGF receptors, neuropilins- 1 and -2 . The primary ligands for the neuropilins are semaphorins, but these receptors also functionally bind multiple VEGF isoforms as well as placental growth factor [23-25]. The neuropilins also serve as coreceptors for c-Met binding to HGF and the PDGF receptors. Both HGF and PDGF are known to be important factors for vascular migration [3,39]. Thus, highly upregulated neuropilin genes found in cultured hMSCs likely play a role in mediating migration of these cells.

HGF, both a cytokine and migration factor plays a key role in vasculogenesis and angiogenesis [39,40]; is also significantly upregulated at sites of tissue damage. Neutralization of HGF signaling has been shown to inhibit wound repair and vasculogenesis [40,41]. HGF is typically secreted by mesenchymal cells and increases motility by epithelial and endothelial cells. The inability of most mesenchymal cells to respond to HGF results from the lack of the c-Met receptor [39]. MSCs are an exception in that they both express genes for HGF and the c-Met receptor [29,43]. Neuss et al., [29] demonstrated HGF inhibited hMSC proliferation, but exerted a strong chemotactic signaling. Migration induced by HGF is different from most other chemokines in that HGF induces cellular motility in a non-directional manner, hence the alternate name, scatter factor [40]. This dual characteristic of hMSCs could induce migration of MSCs in the context of injury. The roles for HGF and neuropilin expression in wounds are currently not well understood, especially since both are expressed by cultured hMSCs [21,44]. More work is needed to better understand the functions of these molecules as produced by MSCs in wounds. 
Based on our experiments the migration of hMSCs in the wound bed does not appear to be fully responsible for the positive effects associated with wound healing such as enhanced granulation tissue formation. Granulation tissue formation develops early and is initiated at the bottom of the wound, at a distance from the advancing hMSCs [13]. Further, hMSCs delivered by the Hyalomatrix PA do not migrate, but still induce earler granulation tissue formation than un-seeded matrix. These observations suggest hMSCs direct the vasculature at a distance, presumably through the production and release of pro-angiogenic factors such as VEGFs, HGF, and PDGFs [23,27,31]. This would also explain positive effects seen when treating wounds with MSC conditioned media containing hMSC derived paracrine factors and exosomes [32,34,45].

Wound healing in rodent skin differs significantly from that in human skin [11,13]. First, healing in rodents is initiated at the base of the skin with the restoration of the subdermal vascular plexus and the initiation of granulation tissue formation that emanates from this structure. Second, rodents possess a thin skeletal muscle sheath, the panniculus carnosum that is located immediately beneath the subdermal vascular plexus. This structure induces the contraction and closure of the wound bed. The hMSCs appear to functionally interact with both of these structures in their promotion of wound healing.

All mice employed in this study were standard immunocompetent animals that did not demonstrate an adverse response to the placement of hMSCs in their wounds. Similar observations have been made in other studies that involve the deployment of either allogeneic or xenogeneic therapeutic cells [4,7]. The placement of xenogeneic cells in a wound environment provides the possibility of interrogating therapeutic cells on the basis of species variance in genomic and proteomic analyses. Wound provides a rich and diverse inflammatory environment that alter the physiological responses of delivered cells. Such environments can only be partially mimicked in culture situations.

An important point demonstrated by this study is that it is possible to deliver significant numbers of hMSCs to murine skin wounds so that these cells remain highly concentrated in the wound. The presence of human cells in a murine background makes it theoretically possible to distinguish the human secretome products and to identify human genome expression in an in vivo inflammatory context. Human cells can be pre-conditioned in culture with proinflammatory agents and analyzed [46]. However, this does not fully replicate inflammatory conditions. The high clustering of fluorescently labelled hMSCs makes it feasible to identify these cells in unstained frozen sections and to selectively retrieve them using laser capture technologies.

\section{Summary/Conclusions}

Human MSCs possess immunomodulatory characteristics that enable them to promote wound healing in both allogeneic and xenogeneic situations. This is demonstrated in the current study where adult human MSCs induced enhanced granulation tissue formation and enhanced repair and function of the mouse panniculus carnosum to contract the wound region. These events result from the release of soluble factors by the applied MSCS. Placement of such cells in a xenogeneic context should provide a methodology with which to better understand the in situ response of these therapeutic cells 


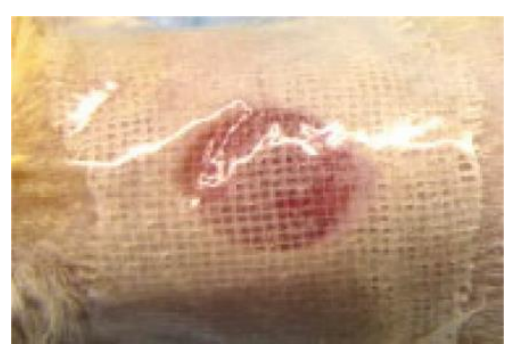

Figure 1. Full-Thickness Cutaneous Wound. An 8-mm wound is created on the dorsal surface. The wound is covered with Vaseline gauze and Tegaderm. A band-aid (not shown) covers the dressed wound.

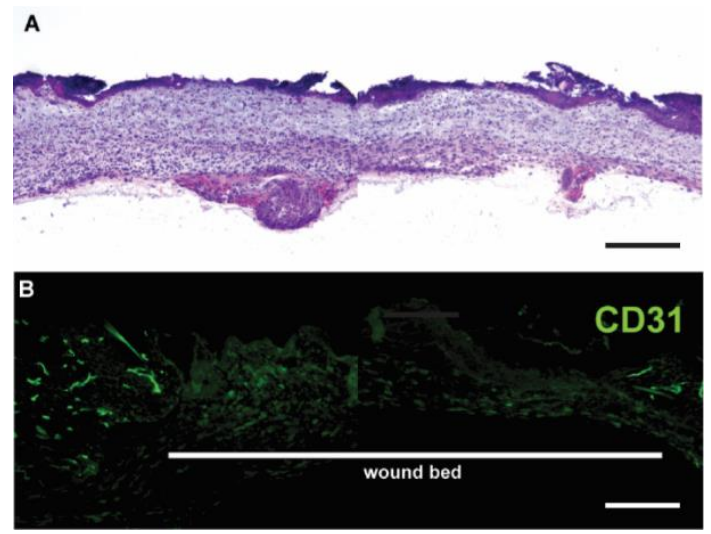

Figure 2. Full-Thickness Cutaneous Wound, Day 2 Post-Surgery. An avascular wound bed has formed, A) Hematoxylin and Eosin, B) CD31/PECAM immunostain. Bars lower right $=500 \mu \mathrm{m}$.

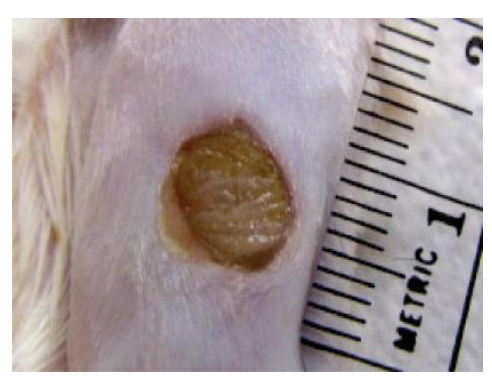

Figure 3. Wound with Carrier, Day 3 Post-Surgery. Carriers with or without hMSCs are placed on the wound bed. The wound is dressed and bandaged as before. 


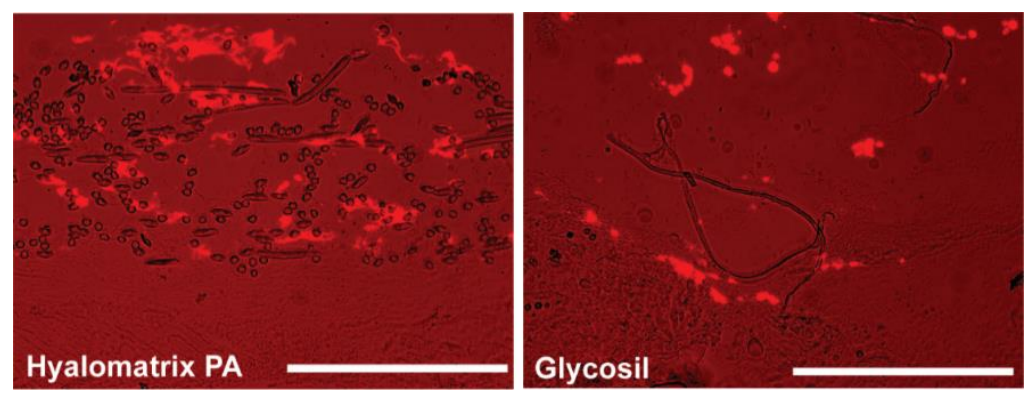

Figure 4. Delivery of MSCs to Wounds, Day 4 Post-Surgery. A comparison of Hyalomatrix PA and Glycosil carriers.

MSCs were labeled with CM-DiI, no other staining of cells and tissues. Bars $=500 \mu \mathrm{m}$
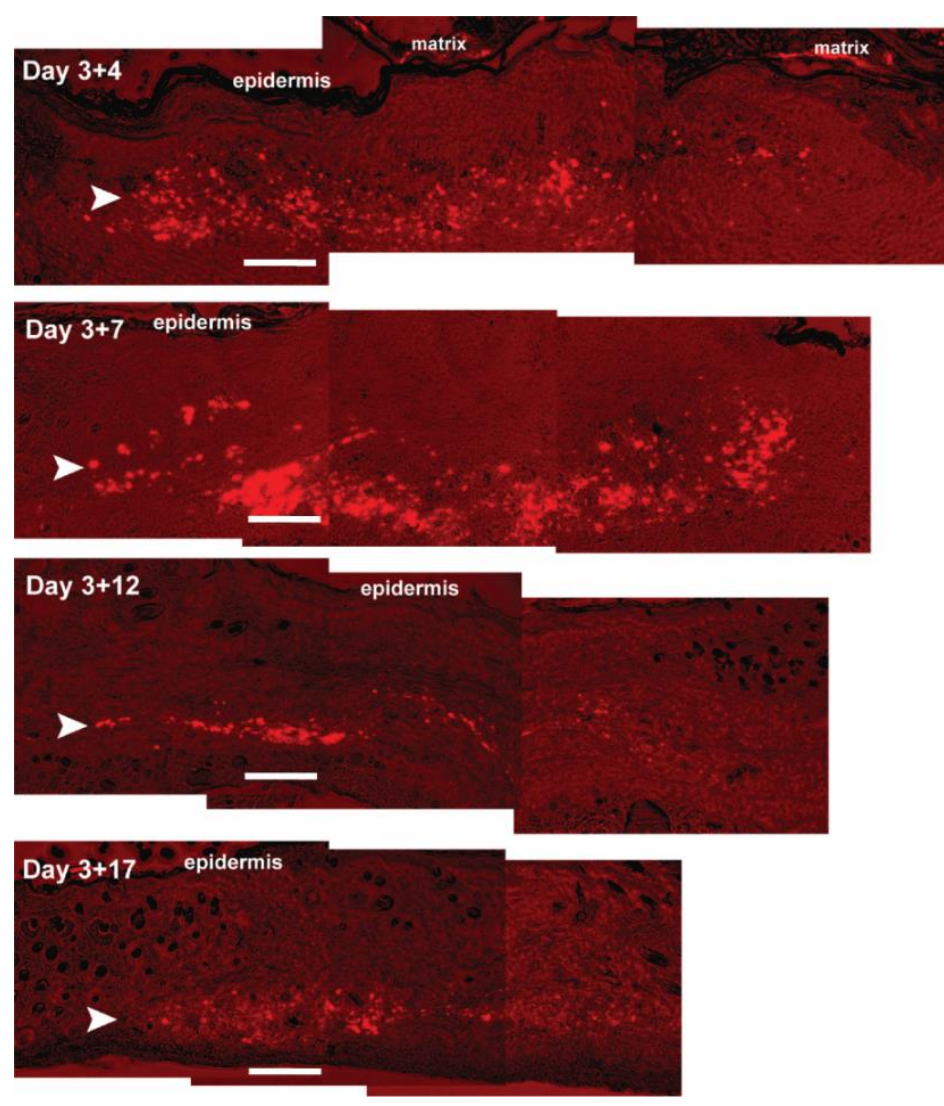

Figure 5. Tracking MSCs into the Wound Bed, Glycosil Carrier Delivery. MSCs were delivered to the wound on day 3 post-surgery and samples were taken at the indicated days post-delivery. MSCs were labeled with CM-DiI, no other staining of cells and tissues. Bars $=350 \mu \mathrm{m}$. 


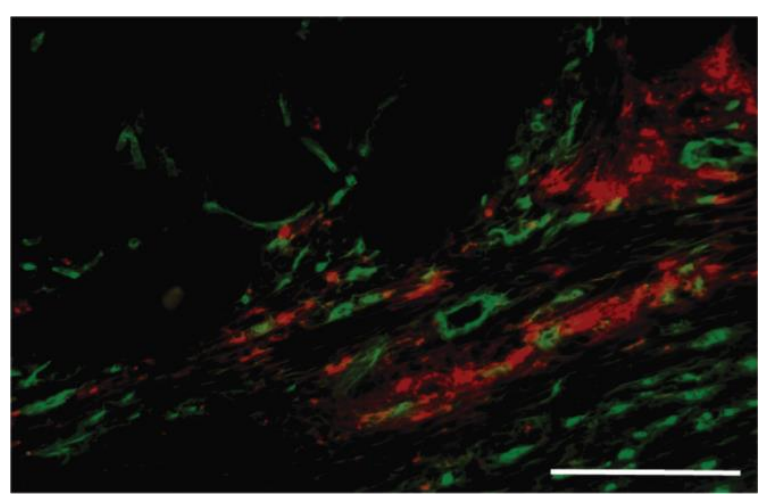

Figure 6. Sub-Dermal Vascular Plexus, Day 20 Post-Surgery. MSCs are red and vasculature labeled with CD31/PCAM is green. Bar $=150 \mu \mathrm{m}$.
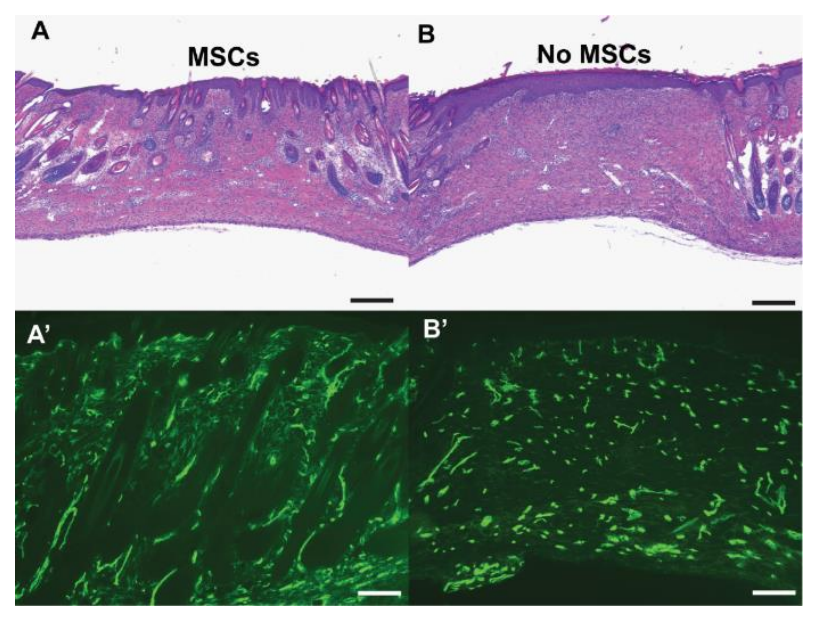

Figure 7. Wound Healing Day 25 Post-Surgery. A comparison is made between a wound that received MSCs in a carrier $\left(\mathrm{A}, \mathrm{A}^{\prime}\right)$ and a wound that received the carrier alone $\left.\left(\mathrm{B}, \mathrm{B}^{\prime}\right) . \quad \mathrm{A}, \mathrm{B}\right)$ Hematoxlin and Eosin; $\left.\mathrm{A}^{\prime} \mathrm{B}^{\prime}\right)$ CD31/PECAM immunostaining. Bars $=300 \mu \mathrm{m}$. 


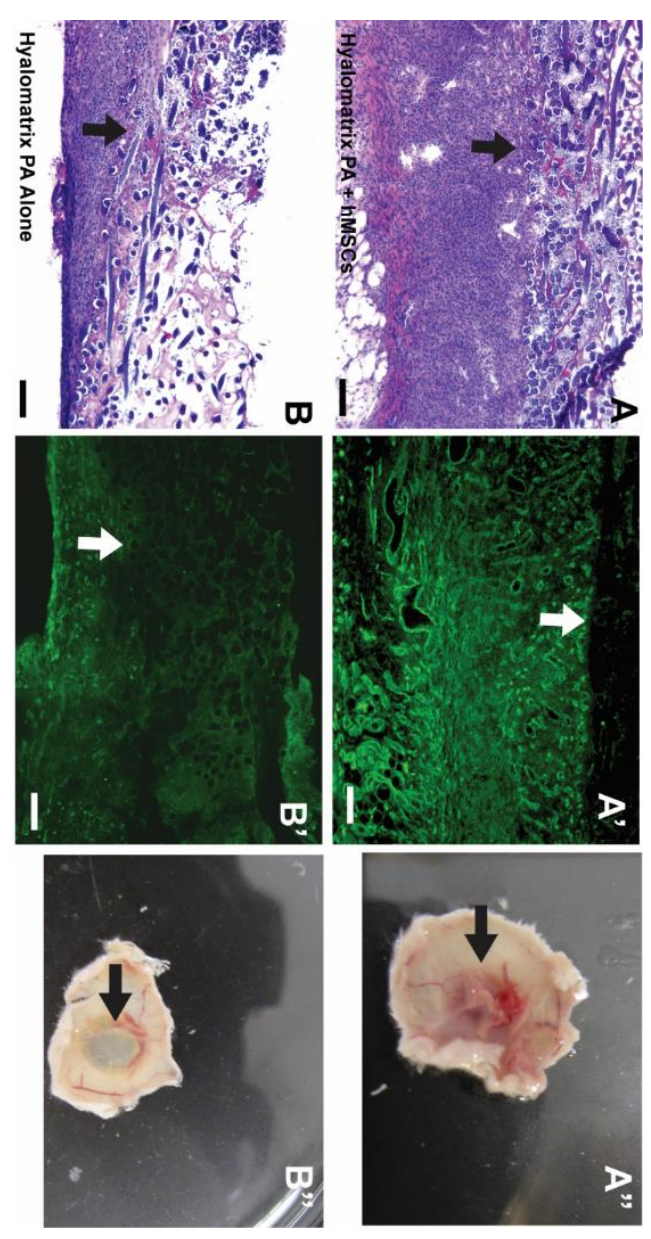

Figure 8. Hyalomatrix PA Delivered to Wound Day 3, A) with and B) without MSCs. A, B) Hematoxylin and eosin stained comparisons. $\mathrm{A}^{\prime}$ ) arrow indicates the top of the wound bed and $\mathrm{B}^{\prime}$ ) the arrow indicates the junction of implant and wound bed, $\left.\mathrm{A}^{\prime}, \mathrm{B}^{\prime}\right)$ CD31/PECAM staining, arrows indicate the junction of the implant and wound bed, $\left.\mathrm{A}^{\prime \prime}, \mathrm{B}^{\prime \prime}\right)$ The bottom of the wounds are shown (arrows). Bars $=200 \mu \mathrm{m}$.

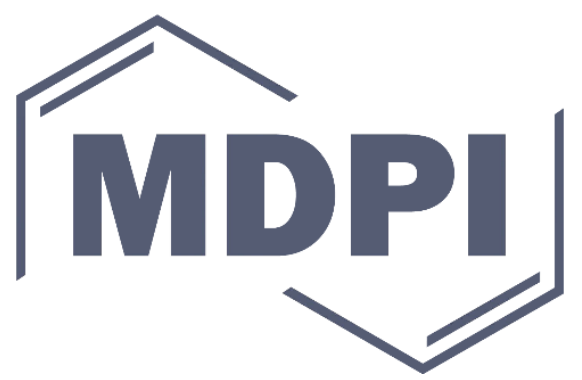

Table 1. RNA-Seq Profiles - Migration Receptors and Angiogenic Factors

\begin{tabular}{|l|l|l|l|l|}
\hline Migration Factors & $\begin{array}{l}\text { Angiogenesis } \\
\text { Factors }\end{array}$ & \\
\hline Gene & RNA-Seq & Gene & RNA-Seq \\
\hline
\end{tabular}




\begin{tabular}{|l|l|l|l|l|}
\hline FLT1/Vegfr1 & 2 & & VEGFA & $29535 \pm 11160$ \\
\hline KDR/Vegfr2 & 5 & & ANPT1 & $1656 \pm 390$ \\
\hline NRP1/neuropilin 1 & $12067 \pm 2180$ & ANGPTL2 & $4982 \pm 1820$ \\
\hline NRP2/neuropilin 2 & $8023 \pm 3160$ & & HGF & $3059 \pm 2140$ \\
\hline MET/ c-Met & $3061 \pm 1360$ & & & \\
\hline PDGFRA/pdgfra & $21170 \pm 12170$ & & & \\
\hline PDGFRB/pdgfrb & $20873 \pm 2410$ & & & \\
\hline
\end{tabular}

This Table provides the average and standard deviation for the indicated genes expressed by three donor populations of cultured hMSCs. Data obtained from Vail et al., [21].

Contributions of Authors. J. Michael Sorrell, Marilyn A. Baber, Rodrigues A. Somoza, Jonathan Kenyon, and Arnold I.

Caplan

JM Sorrell participated in all elements of the project conceptualization, methodology design, and writing of the manuscript. RA Somoza provided resources for RNA-Seq data and assisted in writing. MA Baber supervised and performed human cell culture work, histological studies, and data interpretation. J Kenyon participated in the editing and writing of the manuscript. AI Caplan provided oversight for all aspects of the study and participated in the writing of the manuscript.

Funding: This research was funded by The Armed Forces Institute of Regenerative Medicine grant 4.6.2 for Mesenchymal Stem Cells for Burn and Wound Healing.

The link to the RNA-Seq data repository is as follows: https://www.ncbi.nlm.nih.gov/geo/query/acc.cgi?acc=GSE128554. The GEO accession number is GSE128554.

Acknowledgments: We gratefully acknowledge the gifts of Glycosil ${ }^{\mathrm{TM}}$ from Dr. Glenn Prestwich, University of Utah, Salt Lake City, UT and Hyalomatrix PA ${ }^{\mathrm{TM}}$ from Dr. Alessandra Pavisio, Fidia Advanced Bioplymers, currently a division of Anika Therapeutics, Bedford, MA. Dr. Randall Young provided exceptional assistance with veterinary surgery.

Conflicts of Interest: The authors declare no conflict of interest.

\section{References}

1. Falanga, V.; Iwamoto, S.; Chartier, M.; Yufit, T.; Butmarc, J.; Kouttab, N.; Shrayer, D.; Carson, P. Autologous bone marrow-derived cultured mesenchymal stem cells delivered in a fibrin spray accelerate healing in murine and human cutaneous wounds, Tissue Eng 2007, 13, 1299-1312.

2. Jackson, W.M.; Nesti, L.J.; Tuan, R.S. Mesenchymal stem cell therapy for attenuation of scar formation during wound healing, Stem Cell Res Ther 2012, 3, 20.

3. Karp, J.M.; Teo, G.S.L. Mesenchymal stem cell homing: the devil is in the details. Stem Cell 2009 4, $206-216$.

4. Jeschke, M.G.; Rehou, S.; McCann, M.R.; Shahrokhi, S. Allogeneic mesenchymal stem cells for treatment of severe burn injury. Stem Cell Res Ther 2019, 10, 337. 
5. Chamberlain, G.; Fox, J.; Ashton, B.; Middleton, J. Concise review: mesenchymal stem cells: their phenotype, differentiation capacity, immunological features, and potential for homing. Stem Cells 2007, 25, 2739-2749.

6. Sorrell, J.M.; Caplan, A.I. Topical delivery of mesenchymal stem cells and their function in wounds. Stem Cell Res Ther 2010, 1, 30 .

7. Stoff, A.; Rivera, A.A.; Banerjee, N.S.; Moore, S.T.; Numnum, T.M.; Espinosa-de-los-Monteros, A.; Frichter, D.; Siegal, G.P.; Chow, L.T.; Feldman, D.; Vasconez, L.O.; Mathis, J.M.; Stoff-Khalili, M.A.; Curiel, D.T. Promotion of incisional wound repair by human mesenchymal stem cell transplantation. Exp Derm 2009, 18, 362-369.

8. Rodrigues M, Kosaric N, Bonham CA, Gurtner GC. Wound healing: a cellular perspective. Physiol Rev. 2017, 99, 665706.

9. Martin, P. Wound healing - aiming for perfect skin regeneration. Science 1997, 276, 75-81.

10. Singer, A.J.; Clark, R.A.F. Cutaneous wound healing. New England J Med 1999, 341, 738-746.

11. Wu, Y.; Chen, L.; Scott, P.G.; Tredget, E.E. Mesenchymal stem cells enhance wound healing through differentiation and angiogenesis. Stem Cells 2007, 25, 2648-2659.

12. Wang, X.; Ge, J.; Tredget, E.E. The mouse excisional wound splinting model, including applications for stem cell transplantation. Nature Protocols 2013, 8, 302-309.

13. Galiano, R.D.; Michaels, J.; Dobryansky, M.; Levine J.P.; Gurtner, G.C. Quantitative and reproducible murine model of excisional wound healing. Wound Repair Regen 2004, 12, 485-492.

14. Zomera, H.D.; Trentina, A.G. Skin wound healing in humans and mice: Challenges in translational research. J Dermatol Sci 2018, 90, 3012.

15. Chen, L.; Tredget, E.E.; Wu, P.Y.; Wu, Y. Paracrine factors of mesenchymal stem cells recruit macrophages and endothelial lineage cells and enhance wound healing. PloS One 2008, 3, e1886.

16. Shu, X.Z.; Liu, Y.; Palumbo, F.; Luo Y.; Prestwich, G.D. In situ crosslinkable hyaluronan hydrogels for tissue engineering. Biomaterials 2005, 25, 1339-1348.

17. Price, D.; Das-Gupta, V.; Leigh, I.M.; Navsaria, H.A. A comparison of tissue engineered hyaluronic acid dermal matrices in a human wound model. Tissue Eng 2006, 12, 3001-3011.

18. Gravante, G.; Delogu, D.; Giordan, N.; Montone, A.; Esposito, G. The use of Hyalomatrix PA in the treatment of deep partial thickness burns. J Burn Care Res 2007, 28, 269-274.

19. Lennon, D.P.; Haynesworth, S.E.; Arm, D.M.; Baber, M.A.; Caplan, A,I. Dilution of human mesenchymal stem cells with dermal fibroblasts and the effects on in vitro and in vivo osteochondrogenesis. Dev Dyn 2000, 219, 50-62.

20. Sorrell, J.M.; Baber, M.A.; Caplan, A.I. Influence of adult mesenchymal stem cells on in vitro vascular formation. Tissue Eng Part A 2009, 15, 1751-1761.

21. Vail, D.J.; Somoza, R.A.; Caplan, A.I,; Khalil, A.M. Transcriptome dynamics of long noncoding RNAs and transcription factors demarcate human neonatal, adult, and human mesenchymal stem cell-derived engineered cartilage. J Tissue Eng Regen Med 2019, 14, 29-44.

22. Carmeliet, P. Mechanisms of angiogenesis and arteriogenesis. Nat Med 2000, 6, 389-395.

23. Nagy, J.A.; Dvorak, A.M.; Dvorak, H.F. VEGF-A164/165 and PIGF. Roles in angiogenesis and arteriogenesis. Trends Cardiovascular Med 2003, 13, 169-175.

24. Gu, C.; Rodriguez, E.R.; Reimert, D.V.; Shu, T.; Fritzsch, B.; Richards, L.J.; Kolodkin, A.L.; Ginty, D.D. Neuropilin-1 conveys semaphoring and VEGF signaling during neural and cardiovascular development. Dev Cell 2003, 5, 45-57.

25. Sulpice, E.; Plouët, J.; Bergé, M.; Allanic, D.; Tobelem, G.; Merkulova-Rainon, T. Neuropilin-1 and neuropilin-2 act as coreceptors, potentiating proangiogenic activity. Blood 2008, 111, 2036-2045.

26. Munshi, A.; Mehic, J.; Creskey, M.; Gobin, J.; Gao, J.; Rigg, E.; Muradia, G.; Luebbert, C.C.; Westwood, C.; Stalker, A.; Allan, D.S.; Johnston, M.J.W.; Cyr, T.; Rosu-Myles, M.; Lavoie, J.R. A comprehensive proteomics profiling identifies 
NRP1 as a novel identity marker of human bone marrow mesenchymal stromal cell-derived small extracellular vesicles. Stem Cell Res Ther 2019, 10, 401.

27. Forte, G.; Minieri, M.; Cossa, P.; Antenucci, D.; Sala, M.; Gnocchi, V.; Fiaccavento, R.; Carotenuto, F.; De Vito, P.; Baldini, P.M.; Pra,t M.; Di Nardo, P. Hepatocyte growth factor effects on mesenchymal stem cells: proliferation, migration, and differentiation, Stem Cells 2006, 24, 23-33.

28. Lindblom, P.; Gerhardt, H.; Enge, M.; Hellstrom, M.; Backstrom, G.; Fredriksson, S.; Landegren, U.; Nystrom, H.C.; Bergstrom, G.; Dejana, E.; Ostman, A.; Lindahl, P.; Betsholtz, C. Endothelial PDGF-B retention is required for proper investment of pericytes in the microvessel wall. Genes Dev 2003, 17, 1835-1840.

29. Neuss, S.; Becher, E.; Wöltje, M.; Tietze, L.; Jahnen-Dechent, W. Functional expression of HGF and HGF receptor/c-met in adult human mesenchymal stem cells suggests a role in cell mobilization, tissue repair, and wound healing. Stem Cells 2004, 22, 405-414.

30. Gruber, R.; Kandler, B.; Holzmann, P.; Vögele-Kadleetz, M.; Losert, U.; Fisher, M.B.; Watzek, G. Bone marrow stromal cells can provide a local environment that favors migration and formation of tubular structures of endothelial cells. Tissue Eng 2005, 11, 896-903.

31. Ponte, AL.; Marais, E, Gallay, N.; Lanonné, A.; Delorme, B.; Hérault, O.; Charbord, P.; Domenech, J. The in vitro migration capacity of human bone marrow mesenchymal stem cells: comparison of chemokine and growth factor chemotactic activities, Stem Cells 2007, 25, 1737-1745.

32. Hanson, S.E.; Bentz, M.L.; Hematti, P. Mesenchymal stem cell therapy for nonhealing cutaneous wounds. Plast Reconstr Surg 2010, 125, 510-516.

33. Caplan, A.I.; Correa, D. The MSC and injury drugstore. Cell Stem Cell 2011, 9, 11-15.

34. Hu, P.; Yang, Q.; Shi, C.; Wang, D.; Armato, U.; Dal Prà, I.; Chiarini, A. Mesenchymal stromal cells-exosomes: a promising cell-free therapeutic tool for wound healing and cutaneous regeneration. Burns Trauma 2019, 7, 38.

35. Crisan, M.; Yap, S.; Casteilla, L.; Chen, C.-W.; Corselli, M.; Park, T.S.; Andriolo, G.; Sun, B.; Zheng, B.; Zhang, L.; Norotte, C.; Teng, P.-N.; Traas, J.; Schugar, R.; Deasy, B.M.; Badylak, S.; Bühring, H.-J.; Giacobino, J.-P.; Lazzari, L.; Huard, J.; Péault, B. A perivascular origin for mesenchymal stem cells in multiple human organs. Cell Stem Cell 2008, 3, 301-313.

36. Jain, R.K.; Booth, M.F. What brings pericytes to tumor vessels? J Clin Invest 2003, 112, 1134-1136.

37. Kean, T.J., Lin, P.; Caplan, A.I.; Dennis, J.E. MSCs: delivery routes and engraftment, cell-targeting strategies, and immune modulation, Stem Cells Int 2013, 2013, 732743.

38. Lin, P.; Correa, D.; Kean, T.J.; Awadallah, A.; Dennis, J.E.; Caplan, A.I. Serial transplantation and long-term engraftment of intra-arterially delivered clonally derived mesenchymal stem cells to injured bone marrow, Molec Ther 2014, 1: 160168.

39. Bielmann, M.; Birk, G.; Lenter, M.C. Human primary co-culture angiogenesis assay reveals additive stimulation and different angiogenic properties of VEGF and HGF. Cytokine 2004, 26, 178-185.

40. Birchmeier, C.; Birchmeier, W.; Gherardi, E.; Vande Woude, G.F. Met, metastasis, motility and more. Nat Rev Mol Cell Biol 2003, 4, 915-925.

41. Yoshida, S.; Yamaguchi, Y.; Itami, S.; Yoshikawa, K.; Tabata, Y.; Matsumoto, K.; Nakamura, T. Neutralization of hepatocyte growth factor leads to retarded cutaneous wound healing associate with decreased neovascularization and granulation tissue formation. J Invest Dermatol 2003, 120, 335-343.

42. Sorrell, J.M.; Baber, M.A.; Caplan, A.I. Human dermal fibroblast subpopulations; differential interactions with vascular endothelial cells in coculture: nonsoluble factors in the extracellular matrix influence interactions. Wound Rep Regen 2008, 16, 300-309.

43. Takai, K.; Hara, J.; Matsumoto, K.; Hosoi, G.; Osugi, Y.; Tawa, A.; Okada, S.; Nakamura, S.; Matsumoto, K.; Hosoi, G.; Osugi, Y.; Tawa, A.; Okada, S.; Nakamura, T. Hepatocyte growth factor is constitutively produced by human bone marrow stromal cells and indirectly promotes hematopoiesis. Blood 1997, 89, 1560-1565. 
44. Zacchigna, S.; Pattarini, L.; Zentilin, L.; Moimas, S.; Carrer, A.; Sinigaglia, M.; Arsic, N.; Tafuro, S.; Sinagra, G.; Giacca, M. Bone marrow cells recruited through the neuropilin-1 receptor promote arterial formation at the sites of adult neoganiogenesis in mice. J Clin Invest 2008, 118, 2062-2075.

45. Tang, Y.L.; Zhao, Q.; Qin, X.Y.; Shen, L.P.; Cheng, L.L.; Ge, J.B.; Phillips, M.I. Paracrine action enhances the effects of autologous mesenchymal stem cell transplantation on vascular regeneration in rat model of myocardial infarction. Ann Thorac Surg 2005, 80, 229-236.

46. Ranganath, S.H.; Levy, O.; Inamdar, M.S.; Karp, J.M.; Nehru, J. Harnessing the mesenchymal stem cell secretome for the treatment of cardiovascular disease. Cell Stem Cell 2012, 10, 244-258. 